\title{
SOBRE LA NATURALEZA INTERNA DEL CONOCIMIENTO Y LA IMPOSIBILIDAD DEL ESCEPTICISMO
}

\section{ON THE INTERNAL NATURE OF KNOWLEDGE AND THE IMPOSSIBILITY OF SCEPTICISM}

\author{
Allan González Estrada ${ }^{1}$ \\ Universidad Nacional \\ a.a.gonzalez.estrada@keele.ac.uk \\ Recibido 14 de julio del 2017 / Aprobado 22 de setiembre del 2017 \\ Publicado 7 de noviembre del 2017
}

\section{Resumen:}

Al menos en las tres últimas décadas, la posición representacionalista ha sido el enfoque principal al problema metafísico de la conciencia. Desde ese punto de vista, la explicación de la conciencia, en términos físicos, intencionales y externos, es posible en tanto el cerebro en sí es excluido de una posible fuente de la conciencia. Sin embargo, esta posición es altamente problemática. Ofrezco aquí otra visión, basada en un abordaje internalista de la experiencia e intencionalidad por medio de un fisicalismo no reduccionista, en la que se explica que es el mejor camino para explicar la naturaleza de nuestra consciencia fenoménica e intencionalidad. Esta posición evita el problema del escepticismo, a la vez que es una explicación sencilla de la naturaleza metafísica y epistemológica de la realidad.

Palabras clave: conciencia, intencionalidad, escepticismo, internalismo, externalismo, fisicalismo, estados mentales.

\footnotetext{
Abstract:

Representationalism has been the philosophical mainstream approach to the metaphysical problem of consciousness, at least in the last three decades. Under this view, the explanation

1 Cuenta con un Bachillerato en Filosofía y una Maestría en Bioética, Universidad Nacional - Universidad de Costa Rica. Es egresado de la maestría en Ciencias Cognitivas de la Universidad de Costa Rica. Cuenta con un Doctorado en Filosofía por la Keele University, Staffordshire, Reino Unido. Actualmente es profesor en la Escuela de Filosofía de la Universidad Nacional.
} 
of consciousness in physical, intentional and external terms is possible while the brain itself is excluded as the source of consciousness experience. However, this account is highly problematic. I offer another view, based on an internalistic approach of experience via a non-reductive physicalism, that explain that it is the best path to explain the nature of our phenomenal consciousness and intentionality. This view while avoid the problem of scepticism is a straightforward explanation of the whole metaphysical and epistemological nature of the reality.

Keywords: consciousness, intentionality, scepticism, internalism, externalism, physicalism, brain states.

\section{La metafísica de la conciencia y la naturaleza del conocimiento}

Parece que no dudamos al estar en el supermercado, ante la percepción directa de una manzana ${ }^{2}$, de que esta lo sea, pues el color, el olor, la textura y el sabor nos brindan la certeza de que, efectivamente, hay una manzana. Estas son, quiero afirmar, las propiedades fenoménicas ${ }^{3}$ que registra nuestro cerebro de los datos del mundo exterior ${ }^{4}$ procesados por lo sentidos, y son estas las que experimentamos directamente y, al menos en este caso, las que nos dan la información para creer que hay una manzana real enfrente de nosotros ${ }^{5}$. En otras palabras, se puede argumentar que la existencia del mundo exterior está determinada por la forma en la que nuestra concienciao el carácter fenoménico de la experiencia - es registrada ${ }^{6}$ por el cerebro a partir de los datos del mundo exterior, más que por una conexión directa

2 Esto es, la experiencia que realiza nuestro cerebro al tener una manzana real que excite nuestros sentidos, no es que nuestra experiencia esté dada por una conexión directa con los objetos del mundo, tal como sugieren los representacionalistas como Michael Tye (1995, 2000). Sin embargo, podría ser una completa alucinación, por lo que no sería fácil sostener, a partir de la idea representacionalista, el cómo sería la "experiencia" de la manzana alucinada, o como se determinaría su contenido intencional, aunque Michael Tye trata de establecer una explicación sobre esto no es del todo satisfactoria, particularmente cuando parte del argumento de la transparencia de la experiencia.

3 Llamadas también el carácter subjetivo de la experiencia (Nagel, 1974).

4 Esto se podría poner como la forma en la que, por medio de las cualidades secundarias, nos conectamos con las cualidades primarias, sobre lo cual voy a retornar más adelante.

5 En otras palabras, en este artículo se defiende una posición sobre nuestra percepción basada en un realismo indirecto, o realismo representativo. Aún más, todo podría ser el resultado de un genio maligno al estilo cartesiano y todo lo que se cree conocer no sería más que una completa ilusión. Sin embargo, es plausible suponer que efectivamente existe un mundo exterior, con objetos que, dadas sus características físicas y dada la forma en la que físicamente impactan nuestros sentidos, promueven que toda esa información externa termina con la percepción que en nuestro cerebro se tiene de ellos. Hay estudios empíricos que respaldan esta posición, de que es nuestro cerebro el que construye la realidad del mundo exterior (J. R. Smythies \& V. S. Ramachandran, 2008) (Smythies, 2005).

6 El término realización viene de la idea de múltiple realización de Putnam (1967). 
con el mundo, establecida por un contenido intencional, tal como sugiere Michael Tye $(1995,2000)$. Sin embargo, por un lado, la posición que defiendo podría llevar a un cuestionamiento sobre la existencia del mundo exterior y, por otro, la segunda posición, la representacionalista, nos podría llevar a tener problemas con los contenidos de los sueños y alucinaciones. Pero, más generalmente, la pregunta que subyace es ¿cómo se determina el contenido de la experiencia fenoménica y cómo esta establece la existencia del mundo exterior? Si partimos de la idea de que es en el cerebro en donde se realiza nuestra experiencia, es necesario explorar esta posición internalista, desechar la idea representacionalista ${ }^{7}$ y establecer unas bases claras y distintas que permitan evitar los problemas que la posición por defender podría generar, sobre el escepticismo del mundo exterior ${ }^{8}$. Entonces, es la naturaleza de la conciencia fenoménica lo que vamos a explorar en este artículo. Determinarla se ha vuelto el centro de recientes debates en filosofía, particularmente la forma en la que la conciencia trabaja (McGinn, 1989, Van Gulick, 1990, Tye, 1995, Tye, 2000, Chalmers, 1995, Chalmers, 2013 \& González, 2016), en la que la intencionalidad opera (Tye, 1995 \& Crane, 2003), y cómo ambas están relacionadas con la base física sobre la que opera el cerebro. Es indudable que entender esas relaciones entre conciencia e intencionalidad es lo que nos llevará a un camino diferente para explorar la certeza de que existen objetos externos y la forma en cómo podemos tener ese conocimiento.

\section{El mundo exterior y la experiencia fenoménica}

El cómo nos enfrentamos a un objeto, esto es la percepción, la experiencia y la representación, y con ello cómo se determina el contenido mental del objeto dependerán de la forma en la que podemos entender la naturaleza metafísica de la mente. Por ejemplo, de la misma manera que se puede ver y de ahí creer que hay una manzana en el supermercado, se puede decir yo tengo una mano, pues la veo, la puedo mover, la uso para escribir,

7 Se debe recordar que, de acuerdo con la posición representacionalista, no hay nada en la cabeza relevante para determinar la experiencia fenoménica (Tye, 1995, p. 168).

8 Sin embargo esta posición internalista, podría generar preocupaciones sobre el problema duro de la conciencia (Chalmers, 1995) o el vacío explicatorio (Levine, 1983), pero si se asume una posición metafísica basada en un fisicalismo no reduccionista, ambos problemas podrían solventarse, esto se discutirá más adelante. 
hay un contenido que podríamos llamar proposicional, que aparentemente determina el valor de verdad de mi creencia ${ }^{9}$, y así podemos tener cierto conocimiento. Parece que de eso no hay duda y, sin embargo, ¿podríamos usar ese mismo conocimiento para decir que tanto la mano como la manzana son dos objetos reales, que existen en el mundo? Una forma de determinar el contenido de un estado mental ${ }^{10}$ es asumiendo que este contenido es proposicional. Tal como lo define Byrne: "Algunos estados mentales tienen contenido... y este contenido son proposiciones: objetos abstractos que determinan condiciones de verdad, mundos-posibles" (Byrne, 2005, p. 232). No obstante, ¿qué determina la veracidad de esa proposición? Podríamos ser cerebros en una cubeta y tener los mismos contenidos de verdad; de hecho, tal como lo menciona Wittgenstein, "Que una proposición pueda, en último término, revelarse falsa depende de lo que se considere que es válido para decidir sobre ella” (Wittgenstein, 1969, p. 7). En otras palabras, la creencia de que Rocinante es un caballo tiene el contenido de que Rocinante es un caballo, y la validez de esta proposición depende de la forma en la que se pueda valorar el grado de verdad: como Rocinante es el caballo del Quijote, y el Quijote es producto de la mente de Cervantes, no se podría probar esa verdad, pero, como Brentano afirma, la intencionalidad puede igual representar objetos inexistentes ${ }^{11}$, y esto no sería mayor problema ${ }^{12}$.

9 Este tema -conocimiento y creencia verdadera - no lo voy a tocar a fondo, ya ha sido tratado, entre otros, por Platón en el Teeteto y en el Cratilo, por Chislhom (1957) y Quine (1960).

10 Tal como sugiere Tim Crane "The notion of content belongs within the theory of intentionality. Intentional mental states fall into different kinds: there are hopes, beliefs, fears, desires and so on. All these mental states exhibit what has been called 'aboutness' or 'directedness': they are about or directed on things." (Crane, 2009, p. 454)

11 Brentano usa las palabras "intencional (o mental) inexistencia de un objeto" (Brentano, 1995, p. 68). Siguiendo el consejo de Tim Crane "Entendamos la idea de intencionalidad tan simple como sea posible -es sobre algo" (Crane, 1995, p. 32). En este sentido, como Dale Jacquette sugiere, la idea más básica de intencionalidad en Brentano es que hay un "acto del pensamiento, y un posible objeto del pensamiento" (Jacquette, 2004, p. 101). Sin embargo, la naturaleza metafísica de tales objetos no es clara (Jacquette, 2004, p.101). Tales objetos parecen ser parte de, o pertenecer a un estado psicológico o un acto psicológico, por lo tanto, de acuerdo con Jacquette, "los objetos del pensamiento están contenidos dentro, o pertenecen a actos psicológicos por los cuales son posibles. Esto es conocido como la tesis de la intencionalidad in-existente en la psicología de Brentano" (Jacquette, 2004, p. 101), en otras palabras, esta intencionalidad se refiere a la manera en la que el objeto (real o no) es presentado en el acto psicológico relevante, y solo por medio de este hay una intencionalidad. Ahora, esta intencionalidad es conceptualizada como la marca de lo mental, si Brentano definió o no un dualismo (que luego retoma Chilshom) no se discutirá en este artículo.

12 Igual podría imaginar un unicornio y tener las propiedades fenoménicas relevantes que me indicarían la estructura de ese objeto, no dejaría de ser imaginario, pero, al ser producto de la mente en sus componente fenoménicos e intencionales, sería esa intencionalidad la que pondría límites a la experiencia, y determinaría la existencia o no de ese objeto en el mundo exterior, esto se discutirá más adelante. 
Ahora bien, se puede objetar que tanto el contenido proposicional como las llamadas actitudes proposicionales ${ }^{13}$ son problemáticas (Churchland, 1981), solo un contenido intencional de deseo, creencia o intención tendría este tipo de contenido, de tal manera que, ante la expresión "yo sé que tengo manos", el contenido proposicional estaría definido por la forma en la que se determina el valor de verdad de esa proposición. Sin embargo, ¿cómo se determina el valor de verdad de tal proposición? Una respuesta, del lado representacionalista, estaría dada por Michael Tye: ver mi mano significa tener condiciones óptimas para la percepción (Tye, 1995, p. 101). No obstante, aunque Tye asume esta posición para representaciones perceptuales, al ser la creencia de que yo veo una mano un contenido intencional, sería razón suficiente dada por una conexión directa con el mundo en óptimas condiciones, para dar por sentada la existencia de mi mano. En otras palabras si, de acuerdo con Tye, para tener un contenido intencional que represente la mano, y que este sea parte del mundo ${ }^{14}$, hay que estar en contacto directo con el mundo, y si esto es el caso y causa de nuestras experiencias, ¿cómo podría determinar la verdad de la existencia de mi mano si la estoy viendo en un sueño, o si fuera un cerebro en una cubeta? Esto es, ¿cómo podría establecerse esa representación, y posterior experiencia o carácter fenoménico de la experiencia, a partir de un sueño? En otras palabras, podría ser que fuéramos solo cerebros en una cubeta y que no existiera, por consiguiente, tal mano.

13 De acuerdo con Pete Mandik, una actitud proposicional es "un estado mental tal como una creencia y un deseo que involucra una relación (la actitud) que una persona asocia a un proposición" (Mandik, 2010, p. 95). En otras palabras, para una persona desear un tomate en el supermercado y creer que hay un tomate, tendría que adoptar una actitud de deseo hacia la proposición "quiero comerme un tomate", y una actitud de creencia ante la proposición "hay un tomate en el supermercado".

14 Witggenstein lo expresa del siguiente modo: Así pues, la afirmación "Sé que aquí hay una mano" puede ampliarse del modo siguiente: "Es mi misma mano la que estoy mirando". En ese caso, una persona razonable no dudaría de lo que sé. - Tampoco el idealista; más bien dirá que, para él, no se trataba de la duda práctica, que está descartada, sino que tras la duda práctica todavía yace una duda. -Que esta sea una ilusión se ha de mostrar de un modo distinto (Wittgenstein, 1969, p. 20). Este pasaje es interesante; por un lado, se podría valorar aquí una insinuación del argumento de la transparencia, y, por otro, el uso del lenguaje como moldeador de la experiencia, en efecto, si esta proposición puede corroborarse empíricamente, no debería ser problemática la forma de determinar el valor de verdad, a como el cálculo no permite equivocación; en otras palabras, si como sugiere Wittgenstein "12 x 12 = 144" es una "proposición" tal que "no es posible contraponer la seguridad matemática a la relativa inseguridad de las proposiciones empíricas" (Wittgenstein, 1969, p. 654), entonces no sería posible tener una certeza de la existencia del mundo exterior a partir de proposiciones empíricas tales como "está lloviendo"o "esta es mi mano", no al menos con la forma en la que construimos esas proposiciones y las valoramos, con las herramientas metafísicas sobre las que descansa la ciencia actual. 
Esta última observación nos lleva, nuevamente, a un enfoque diferente del problema del escepticismo, o quizás a un enfoque que no se había valorado antes: ¿cómo se relaciona nuestro conocimiento del mundo con la experiencia e intencionalidad, y estas con la base física que las realiza?

\section{Dretske y el cierre epistémico.}

Tener una creencia, y que esta sea verdadera, de que hay tomates en el supermercado puede partir de varias bases. Por un lado, puede fundarse de la experiencia previa de que ahí hemos encontrado tomates y, por otro, de la creencia de que con base en esa experiencia hay efectivamente tomates en el supermercado. Otras creencias con contenido de verdad son más difíciles de corroborar. Por ejemplo, que mañana salga el sol, o que no soy un cerebro en una cubeta no son, al menos empíricamente, posibles de comprobar, salvo por algún método de inducción que no es, en todo caso, concluyente para tener una comprobación verdadera.

Una primera aproximación a la forma en la que conocemos hechos del mundo, esto es, a cómo podríamos saber que nuestras creencias son verdaderas, parte de las ideas de Fred Dretske, quien, de acuerdo con Matthias Steup, sostiene que "conocer $\mathrm{p}$, requiere tener una razón conclusiva para p" (Steup, 2005, p. 1). Según Dretske, este principio se establece de la siguiente manera: "Suppose, then, that (I) S knows that P and he knows this on the basis (simply) of R, entails (2) R would not be the case unless $\mathrm{P}$ were the case" (Dretske, 1971, p. 1). En otras palabras, si S sabe que hay tomates en el supermercado, lo conoce con base en su experiencia, por lo que no tendría ese conocimiento si no tuviera esa experiencia, que le da a la creencia un valor de verdad. Ahora, esta razón conclusiva es, en términos de Dretske, un contrafáctico, según Steup, "una declaración sobre lo que debería (o no debería) ser el caso, si las cosas fueran diferentes de lo que de hecho son" (Steup, 2005, p. 1). Pero, ¿cómo una lógica contrafáctica puede determinar el conocimiento? Dretske lo que trata de buscar son justificaciones empíricas para nuestro conocimiento. Si este se encuentra determinado por los contenidos objetivos de los objetos del mundo exterior, para Dretske la forma en la que conocemos esos objetos será por medio de la intencionalidad o representación en términos de información, de cómo están determinados 
externamente (Dretske, 1981). Y este conocimiento externo es el que nos da la ciencia de las cualidades primarias. En otras palabras, ante la experiencia visual (A) de ver un tomate en el supermercado (B), la persona que lo observa conoce (B) solo su razón para creer en (B) es conclusiva para (A), lo cual solo tendría que cumplir con una valoración de un contrafáctico, tal como lo explica Dretske: si no hay (B), la persona no tendría (A).

Por supuesto, es necesario resaltar la posición de Dretske, la cual está relacionada con la metafísica de la conciencia que él establece, dicho de otro modo, con la idea de que lo que representamos está en el mundo exterior, y no en nuestra cabeza (Drestke, 1995, p. 75). Por lo tanto, se puede concluir que una teoría del conocimiento, de acuerdo con Dretske, es posible solo enfatizando el hecho de que tenemos un contacto directo con el objeto. Pero, ¿y si es una ilusión? ${ }^{15}$ Si este fuera el caso, Dretske podría argumentar que no hay contenido intencional, el objeto no está afuera de la cabeza, no estamos en un contacto directo, y no hay contenido fenoménico dado por el contenido intencional, por lo que el conocimiento de un objeto alucinado no es posible. No podría hablarse, entonces, de la transparencia de la experiencia, base del representacionalismo, por lo que no nos diría nada del mundo en todo caso. En conclusión, esa forma de establecer el conocimiento del mundo, elaborada por Dretske, no es posible de sostener ${ }^{16}$; las alucinaciones y sueños tienen contenido intencional y fenoménico, en otras palabras, la naturaleza de nuestro conocimiento del mundo exterior está

15 Chilshom trata de establecer una forma de percibir, sin embargo, parte de dos premisas: una, que la intencionalidad, a partir del análisis de contextos intensionales (con s), puede ser analizada lingüísticamente, y la segunda, que percibir es ver de cierta forma -y, esto es de una manera adverbial-, en otras palabras, en lugar de ver rojo, de acuerdo con Chilshom, diría que observo algo rojamente. En el caso de la alucinación, el autor establece un error en la apariencia de las cosas, y tiene sentido, puesto que defiende una suerte de realismo directo. Sin embargo, como menciona Kriegel, la intencionalidad no es un asunto lingüístico (Kriegel, 2011, p.126). Esta posición adverbial tiene los mismos problemas con los contenidos de las alucinaciones y Chilshom no lo resuelve adecuadamente, puesto que sigue apelando a un análisis lingüístico, tal como él menciona "...cuando percibimos algo, expresamos nuestra percepción en oraciones, donde las cosas percibidas son designadas -o indicadas solamente por términos demostrativos" (Chilshom, 1957. p, 163)

16 Es más, Dretske luego asume que esta forma de determinar el conocimiento no puede ser correcta, por ejemplo, ante la proposición "Elisa se comió los elotes" se establecen ciertas proposiciones que, como afirma Steup, no parecen ser posibles de determinar: "Hay objetos físicos, hay otras mentes, y el pasado es real" (Steup, 2005, p. 3). En otras palabras, y según Wittgenstein, como empíricamente podemos probar la proposición "el pasado es real", no tienen estas palabras el grado de certeza que podemos tener de una proposición tal como 5 x $5=25$. Esto supone ya un problema para conocer el mundo a partir de proposiciones, o dicho de otro modo, no podría sostenerse que el contenido de un estado intencional es estrictamente proposicional, es más, podría argumentarse que los estados perceptuales no tiene contenido conceptual y, por ende, proposicional, en esto estaría de acuerdo con Tye: no se necesita el concepto de rojo, para tener una experiencia del rojo (Tye, 1995, p. 139). 
más bien determinada por una realización interna, tanto de la experiencia como de la representación, e internamente significa que es llevada a cabo por estados cerebrales. Lo anterior implica una nueva forma de determinar la manera de evaluar el conocimiento del mundo exterior, a partir de nuestra conciencia e intencionalidad.

\section{La posibilidad interna del conocimiento}

Ahora bien, si la experiencia del mundo no es resultado de un contacto directo con este y más bien es realizada internamente, ¿cómo podemos conocer el mundo exterior? Se puede argumentar que el conocimiento del mundo es un producto de la percepción directa de nuestra experiencia fenoménica -realizada por un estado cerebral ${ }^{17}$ - y que indirectamente conocemos los objetos del mundo ${ }^{18}$. Conocer los objetos del mundo implica tener una experiencia fenoménica que permita representarlos, por lo que es necesario, primero, tener una fundamentación metafísica de la conciencia en términos físicos del cerebro y, luego, determinar cómo esta conciencia genera la representación, esto es, ir de lo interno a lo externo. No es el objetivo de este artículo entrar en este debate metafísico, pero sí mencionar que la mejor opción, en el bazar del problema mente-cuerpo, es el fisicalismo no-reduccionista ${ }^{19}$. Esto es, la experiencia es realizada por estados cerebrales, pero no reducibles a las propiedades físicas que las generan ${ }^{20}$. Ahora bien, tal como indica el argumento del conocimiento de Dretske, si la proposición "yo tengo manos" es verdadera, podría concluirse que no se es un cerebro

17 Si hay una relación directa entre el cerebro y la conciencia, no puede ser reduccionista, esto lleva a un callejón sin salida en la apuesta fisicalista, la naturaleza metafísica de la conciencia debe basarse en un materialismo no reduccionista, tal como lo intuyeron Putnam y Fodor. Ese funcionalismo fisicalista debe descansar en una base tal como la determinan Armstrong (198) y Lewis (1972), no voy a explorar este asunto en este artículo.

18 Esta posición, llamada realismo indirecto, o realismo representativo, ha sido favorecida por recientes investigaciones científicas (Smithies, 2005, Ramachadran, 2008)

19 Los argumentos de Putnam (1967) y Fodor (1974), además de los aportes de Davidson (1972) y Armstrong (1980), nos dan una buena fundamentación para favorecer el fisicalismo no-reduccionista.

20 Si el argumento de Papineau, de la completitud de la física, es correcto, entonces se puede concluir que cualquier proceso físico es resultado de un proceso físico anterior, como el mismo Papineau menciona. Este argumento es suficiente para desterrar cualquier rescoldo dualista (Papineau, 1993, p. 8). En otras palabras, si la conciencia aparece como resultado físico en el cerebro, tiene que ser física, por lo que el fisicalismo no-reduccionista no podría equipararse con el dualismo de propiedades, establecido por Strawson $(1959,1961)$. El fisicalismo no reduccionista es una fuerte alternativa fisicalista para explicar la conciencia sin tener los problema del reduccionismo de Smart (1959), Place (1956), los conflictos eliminativos de Dennett (1991) o el panpsiquismo de Chalmers (1996, 2013, 2015). 
en una cubeta, sin embargo, a partir de una propuesta internalista, el saber que "tengo manos" parte, primero, de la experiencia de ver mis manos y, segundo, de la representación que, a partir de esa experiencia, determina los límites de lo que está en el mundo exterior ${ }^{21}$. Y no parece haber forma de probar empíricamente que somos cerebros en una cubeta, no obstante, si como Wittgenstein nos indica, la posibilidad de conocer la verdad de proposiciones empíricas es incierta, no hay posibilidad de saber que tengo manos, o saber que no soy un cerebro en una cubeta. Sin embargo, si partimos de una posición internalista, tal como lo indica Pautz: "la conciencia primero" (Pautz, 2013, p. 180), entonces al menos podemos establecer de alguna manera la posibilidad de conocer los objetos del mundo exterior -si entendemos correctamente la función y el papel que juega la conciencia -. En otras palabras, si partimos del conocimiento de los objetos desde la experiencia fenoménica, entonces esta, que es directa, nos da un conocimiento indirecto de los objetos del mundo exterior. Ahora bien, esta posición puede generar preocupaciones sobre el conocimiento del mundo exterior, sin embargo, tal como menciono en mi tesis de doctorado:

But I am not agreeing with the idea that there are mental images, or sense data; rather there are phenomenal properties that we are aware of in our perception of an object. And yet, even though phenomenal properties can be explained in terms of a non-reductive physicalist ontology, such properties are seen by some philosophers as highly problematic; some philosophers may think that these 'entities' lead to a scepticism. But this is irrelevant according

21 Como un argumento a favor de esta visión internalista, se puede mencionar la idea de cómo la experiencia es realizada por un estado cerebral, en otras palabras, si, como el representacionalismo clama, nuestros estados cerebrales representan los cambios en el ambiente por medio de una variación causal (Tye, 1995, p. 168), podría pensarse que, en un cerebro en una cubeta, la representación viene dada por los inputs de computadora que se conectan al cerebro. Así, por un lado tenemos una representación producto de un contacto directo con el objeto, o bien, por otro, una representación dada por un input de computadora; si el estado cerebral co varía con estos inputs, entonces la entrada de información es irrelevante, y lo que es relevante es la realización de la experiencia -y la representación, por medio del estado cerebral. De hecho, Tye no niega la posibilidad de un cerebro en una cubeta, solo niega que tenga experiencias perceptuales adecuadas, dado que las experiencias del cerebro en una cubeta son un caso de mala representación. Sin embargo, esto es claro en el caso de Tye, puesto que, como representacionalista, espera un contenido intencional y fenoménico dado por eventos externos, para evitar el vacío explicatorio (Levine, 1982) y el problema duro de la conciencia (Chalmers, 1996). Pero esta posición representacionalista no los resuelve, no al menos con una explicación de cómo la experiencia sería representacional en términos de eventos internos. La posición más adecuada con esto es expresada por Kriegel (2002), sin embargo, no abandona el representacionalismo como fundamentación metafísica de la conciencia. 
to the view that I am defending - perhaps we are brains in vats, but since we have no positive reasons to think we are, this possibility can be dismissed (González, 2016, p. 144).

En otras palabras, solo partiendo de las propiedades fenoménicas, de la realización de las cualidades secundarias, podemos, desde de la representación o intencionalidad, develar las propiedades primarias y estudiarlas objetivamente mediante la ciencia, lo cual determinaría el conocimiento científico del mundo exterior. A partir de un fisicalismo no-reduccionista se puede asegurar que tanto la conciencia como la intencionalidad responden a una apuesta que no necesita más verdades metafísicas, y no pueden esperarse más descubrimientos físicos que las expliquen. El problema duro de la conciencia o el vacío explicatorio no parten solo de una pregunta mal planteada. Es nuestra fenomenología la que nos revela las cualidades secundarias del mundo y, por medio de la intencionalidad, que está determinada por esta fenomenología, se develan las propiedades primarias del mundo. Menciono una de las conclusiones de mi tesis, la cual afirma que el conocimiento del mundo puede ser resultado de un proceso interno, liderado por la fenomenología, en el que el escepticismo no es más que una respuesta natural a una metafísica pasada de moda:

If the idea to explain consciousness is to insist upon a reductive approach, this idea will not allow us to move forward in the relevant explanations of the mind, neither in the philosophical or scientific arena. But we should not be insisting on a reductive approach if we take seriously the problems for reductionism raised by philosophers like Putnam and Fodor. Many still think that the path science must take is to try to explain our conscious experience in terms of more fundamental principles, but that is where they are wrong. They start with the primary qualities and try to explain such qualities first, then they move onto the mind and its phenomenal aspects, thinking these will be explained in terms of such primary qualities. But such explanations will not succeed, since they are rooted in the view that what determines almost everything is our understanding of the primary qualities, so we should try to produce all possible explanations in terms of such qualities. They are right to an extent, since science progresses from the primary qualities which intentionality reveals to us, but they forget where our understanding of 
primary qualities ultimately came from: experience! Hence, they have things the wrong way around. As I have pointed out, the desire for such reductive explanations will make some philosophers think that a physicalist account of consciousness cannot be true, and some scientists think they have to discover something completely unprecedented in order to solve the problem (that there needs to be a 'breakthrough') - this side is pushed by reductionism towards dualism (or some other alternative metaphysics to physicalism) or the belief in the need for a scientific revolution. This wastes the time of both philosophers (dealing with obsolete metaphysics) and scientists (looking for a breakthrough that will never happen). And such breakthroughs will never happen because it is not possible to explain experience of secondary qualities in terms of such primary qualities. In other words, we cannot explain the experience of red in terms of the kind of physical explanation that has been used to describe what is reflected by a given surface, or by measuring the speed of a ball in a free fall, or to describe the laws that govern the movements of the planets. Such explanations describe, as has been explained, primary qualities. And yet to discover what such primary qualities are, it is required to understand first how precisely our minds, in terms of a phenomenal-intentional structure, reveal such primary qualities. This is the alternative that I offer: starting with our phenomenology, and understanding intentionality in phenomenal terms. Otherwise, we will remain held in the grip of reductivism, and so we will be endlessly inspired to reconsider dualism, or even worse, eliminativism (González, 2016, p. 282).

Dado lo anterior, el conocimiento del mundo exterior no se obtiene por una conexión directa con el mundo, no es que el contenido intencional sea el que determine el contenido fenoménico. Más aun, la posición de filósofos como Dretske no es adeccuada en tanto sigan ese camino externalista. Si bien es cierto que determinar la naturaleza de la conciencia en términos de un proceso interno cerebral ha generado una serie de debates a nivel filosófico y en el científico, es el camino correcto para determinar la metafísica de la conciencia y la naturaleza del conocimiento. El reduccionismo, como apuesta metodológica, ha fallado, el pansiquismo, como alternativa, ha generado muchos problemas, a pesar de su popularidad, por lo que la opción, como se ha insinuado en este trabajo, es un fisicalismo no reduccionista. Este nos permite no solo entender la naturaleza interna de la conciencia, sino establecer cómo las cualidades secundarias nos develan las 
primarias, y de esta manera representar y conocer el mundo exterior. Es así que, cuando busco el tomate en el supermercado, espero ver un objeto real, pero lo que percibo estará determinado por nuestra conciencia fenoménica $\mathrm{y}$, en consecuencia, solo indirectamente el conocimiento del mundo exterior es posible, y únicamente podría conocer el tomate y terminar preparando la salsa boloñesa para mi pasta.

\section{Referencias}

Armstrong, D. (2002). The Causal Theory of the Mind. En D. Chalmers. (Ed.), Philosophy of mind: classical and contemporary readings (80-87). Oxford: Oxford University Press. Armstrong, D. (1986). The Nature of Mind. En J. P. Bratman (Eds.), Introduction to Philosophy: Classical and Contemporary Readings (259-266). Oxford: Oxford University Press.

Bókkon, I., Mallick, B. N., \& Tuszynski, J. A. (2013). Near death experiences: multidisciplinary hypothesis. Frontiers in Human Neuroscience, 7, 533. http://doi.org/10.3389/ fnhum.2013.00533

McLaughlin, B., Beckermann, A., \& Walter, S. (2009). The Oxford Handbook of Philosophy of Mind. Oxford: Oxford University Press.

Brentano, F. (1995). Psychology from an Empirical Standpoint. New York: Routledge.

Chalmers, David J. (1995). Facing up to the problem of consciousness. Journal of Consciousness Studies 2 (3):200-19.

Chilshom, R. M. (1957). Perceiving: A philosophical Study. Ithaca: Cornell University Press.

Crane, T. (2001). Elements of Mind. New York: Oxford University Press.

Crane, T. (2009). Is Perception a propositional attitude? The Philosophical Quarterly, 59: 452-469. doi:10.1111/j.1467-9213.2008. 608.x

Davidson, D. (1987), Knowing one's own mind. Proceedings and Addresses of the American Philosophical Association 60 (3):441-458.

Dennett, D. (1991). Consciousness Explained. New York: Penguin.

Dretske, F. (1995). Naturalizing the Mind. Massachusetts: MIT Press.

Dretske, F. (1995). Naturalizing the Mind. Cambridge: MIT Press.

Fodor, J. (1974). Special Sciences (Or: the disunity of science as a working hypothesis). En D. Chalmers, Philosophy of Mind: classic and contemporary readings (126-135). Oxford: Oxford University Press.

Gonzalez Estrada, A. (2016). On the phenomenal, intentional and physical nature of the mind. $\mathrm{PhD}$ Thesis, publicación en preparación, Keele University.

Gulick, R. V. (2002). Nonreductive Materialism - Still the Best Buy at the Mind-Body Bazaar. En M. Pauen(Ed.). Phaenomenales Bewustsein: Entstehung und Erklarg (297327). Berlin: Mentis Verlag.

Gulick, R. V. (1992/1997). Understanding the phenomenal mind: are we all just armadillos? Part II: the absent qualia argument. En O. F. Ned Block, The nature of consciousness (435-442), Massachusetts: MIT Press.

Jackson, F. (1982). Ephifenomenal Qualia. Philosophical Quarterly 32: 127-136. 
Kriegel, U. (2002). Phenomenal Content. Erkenntnis 57(2) 175-198.

Kriegel, U. (2011). The Sources of Intentionality. Oxford: Oxford University Press.

Lewis, D. (1991). Mad Pain and Martian Pain. En D. R. (Ed.), The Nature of Mind (229235). Oxford: Oxford University Press. Trabajo original publicado en 1980.

McGinn, C. (2002). Can we solve the mind-body problem? En O. F. Ned Block, The nature of consciousness Philosophical dabates (529-542). Massachussets: MIT Press. Trabajo original publicado en 1989.

McGinn, C. (1983). The subjective view. Oxford: Claredon Press.

Papineau, D. (1993). Philosphical Naturalism. Oxford: Blackwell.

Pautz, A. (2013). Does Phenomenology Ground Mental Content? En U. Kriegel (Ed.), Phenomanel intentionality (194-234). Oxford: Oxford University Press.

Place, U. (1970). Is consciouness a brain process? En C. V. Boors (Ed.), The mind-brain Identity Theory (42-51). London: The Macmillan Press. Trabajo original publicado en 1956.

Putnam, H. (1975). Mind and Machines. En H. Putnam, Philosophical papers Vol. 2 (362385). Cambridge: Cambridge University Press. Trabajo original publicado en 1960.

Smart, J. (1970). Sensations and brain process. En C. Borts, The Mind-Brain Identity Theory (págs. 52-66). Surrey: The Gresham Press. Trabajo original publicado en 1959.

Smythies, J. (2005). How the brain decides what we see. Journal of the royal society of medicine 98(1): 18-20.

Smythies J. R. \& Ramachandran, V. S. (2008). An empirical refutation of the direct realist theory of perceptio. Inquiry, 437-438.

Strawson, P. (1981). Individuals. En D. Rosenthal(Ed.), The nature of mind (págs. 58-62). London: Methuen \& Co Ltd. Trabajo original publicado en 1958.

Strawson, P. (1991). Person. En D. Rosenthal, The nature of mind (págs. 104-115). Oxford: Oxford University Press. Trabajo original publicado en 1958.

Tye, M. (2000). Consciousness, colour and content. Cambridge, MA: MIT Press.

Tye, M. (1995). Ten problems of consciousness. Massachusetts: MIT Press.

Wittgenstein, L. (2006). Sobre la certeza. Barcelona: Gedisa. Trabajo original publicado en 1969. 